\title{
Analysis of control and sensing interfaces in a Photonic Integrated Chip solution for Quantum Computing
}

\author{
Luca Gemma* \\ Department of Industrial Engineering, University of \\ Trento, I-38123 Povo, Italy \\ luca.gemma@unitn.it \\ Mher Ghulinyan \\ Centre for Materials and Microsystems, Fondazione Bruno \\ Kessler, I-38123 Povo, Italy \\ ghulinyan@fbk.eu
}

\begin{abstract}
Interest in quantum computing is rapidly growing in the scientific community as such technology could be the key to enable intensive use of machine learning and big data algorithms. Quantum computers can be conceived from basic photonic elements, such as Mach-Zehnder interferometers (MZI), but they still need control mechanisms and sensing elements from traditional VLSI technology. In this study, we present an ongoing study on a potential architecture for these basic photonic circuits. We realized two Photonic Integrated Circuit (PIC) test structures embedding metallic thermistors as phase shifters and silicon photodiodes as output detectors. We present a complete characterization of the phase shifter elements for an effective on-chip PIC interaction. To induce a consistent phase shift still retaining coherence of light, we act on the phase-shifters by means of a closed-loop control. By controlling through Pulse-Width Modulation the phase shifters, we drive the path of photons accurately in the PIC, demonstrating the effectiveness of the proposed configuration for the management of a photonic chip.
\end{abstract}

\section{CCS CONCEPTS}

\section{- Hardware $\rightarrow$ Quantum computation. KEYWORDS}

Quantum computing, Photonic Integrated Chips, thermistors, photodetectors

\section{ACM Reference Format:}

Luca Gemma, Martino Bernard, Mher Ghulinyan, and Davide Brunelli. 2020. Analysis of control and sensing interfaces in a Photonic Integrated Chip solution for Quantum Computing. In 17th ACM International Conference on Computing Frontiers ( $C F$ '20), May 11-13, 2020, Catania, Italy. ACM, New York, NY, USA, 4 pages. https://doi.org/10.1145/3387902.3394034

${ }^{*}$ Corresponding author

Permission to make digital or hard copies of part or all of this work for personal or classroom use is granted without fee provided that copies are not made or distributed for profit or commercial advantage and that copies bear this notice and the full citation on the first page. Copyrights for third-party components of this work must be honored

For all other uses, contact the owner/author(s).

CF '20, May 11-13, 2020, Catania, Italy

(c) 2020 Copyright held by the owner/author(s).

ACM ISBN 978-1-4503-7956-4/20/05.

https://doi.org/10.1145/3387902.3394034

\author{
Martino Bernard \\ Centre for Materials and Microsystems, Fondazione Bruno \\ Kessler, I-38123 Povo, Italy \\ bernard@fbk.eu \\ Davide Brunelli \\ Department of Industrial Engineering, University of \\ Trento, I-38123 Povo, Italy \\ davide.brunelli@unitn.it
}

\section{INTRODUCTION}

October 23rd, 2019: Google reaches quantum supremacy. In 200 seconds, Sycamore, Google's quantum computer, resolves an algorithm which would have taken Summit, the fastest non-quantum computer, two days to resolve [1]. Both Google and IBM try respectively to consolidate and to demolish the announced quantum supremacy and, as yet, many scientists do not consider quantum supremacy as "reached". However, following the comparison made by computer scientists, "the first airplane flew for no more than 12 seconds, still since then man has started flying" [2]. Without a doubt, that test of computational strength was a great show of how fast and significant quantum computing is becoming, and, among several possible solutions, quantum photonics is the most promising to have quantum computers replacing conventional machines on our desktops.

Quantum photonics relies on quantum states of photons, the unitary particles of light, to implement the two basic properties of quantum computing: superposition and entanglement. Superposition is reached when, in the quantum scale, two or more states co-exist, while entanglement implies that when two unitary particles are created after a specific event in time, they cannot change their properties independently: no matter how far away they are, as soon as one of them changes, the second particle instantly experiences that change. In a photonic circuit, the path of light is modified by exploiting the thermo-optic properties of the material used as a waveguide, and this change is usually induced with heaters or electro-optic effect. The effect of the heaters is to change the speed of light within the material, thus allowing to change the phase of the photons travelling in the heated waveguide. The control of phase, together with beam-spliting elements, allow for the realization of any unitary transformation of arbitrary states. The output light is then caught with single-photon avalanche diodes (SPADs), which generate an electric signal proportional to the intensity of the incident light.

The effectiveness of quantum photonics in realizing quantum computers has been proved by several works, where different principles and main components were characterized. Annoni et al. [3] inspected a photonic switch that used a common basic building block, namely the MZI, for photonic circuits. They realized a matrix of MZIs and automatically routed these elements between consecutive stages, allowing simultaneous acquisition of a high amount of 
parallel data. Zhu et al. [4] exhaustively investigated phase-shifters used in quantum photonic circuits and demonstrated state-of-theart performance for thermo-optic phase shifters applied to MZIs by using Aluminum Nitride (AIN) between the heaters and the waveguide. In [5], Gentile et al. combined previous knowledge on MZIs to build and test a Bayesian phase estimator, proving how robust these basic blocks are in conjunction with these estimators against most of the common noise sources. Still, in none of these works, the authors characterized thermistors and photodiodes as fundamental elements of a PIC circuitry.

In fact, although many examples of quantum photonic circuits exist (mainly enclosed in the architectures shown in [3], [4] and [5]), little work has been done to fully characterize and inspect, for example, the readout circuit of such chips, thus not allowing a precise output measurement.

In this work, we fill this gap providing a comprehensive characterization of the interfaces between PIC and standard VLSI, and proposing resistive Titanium-Titanium Nitride (Ti-TiN) thermistors, a possible solution for implementing phase shifters in a Photonic Integrated Chip (PIC) made by basic photonic elements such as MZIs. This work is organized as follows: in Section 2 we present a brief overview on quantum photonic chips. Section 3 illustrates our architecture. In Section 4 we discuss our results, focusing on resistive thermistors. Finally, in Section 5 conclusions are drawn.

\section{OVERVIEW}

The possibility to fabricate quantum circuits in a CMOS-like process and the fact that Silicon Nitride (SiN) waveguides can be used within this platform, have established a state-of-the-art for SiN photonic chips [6]. Moreover, the capability of combining monolithically different building blocks in the fabrication process paved the way for developing complex functions on a quantum chip. A building block of particular interest is the Mach-Zehnder interferometer. In its most common configuration, a single MZI is composed of two beam splitters and two phase shifters deployed on two arms. Firstly, a beam splitter divides the two input beams (In A and In B in Figure 1) following a 50-50 power split (for an ideal beam splitter). Secondly, each phase shifter induces a phase delay in the path of light, thus modifying the incoming light in each path. Finally, after passing through a second beam splitter, the two light beams exit (Out A and Out B in Figure 1). As designed in our architecture, the MZIs will have three basic functions depending on the amount of light transferred between each arm: 50/50 beam splitter, full transfer or no transfer of incoming light. The induced phase shift will be produced by exploiting the thermo-optic effect on the SiN waveguides and the thermal gradient will be controlled by the aforementioned Ti-TiN thermistors.

\section{THE ARCHITECTURE}

The two integrated test structures (one embedding thermistors as phase shifters and one with silicon photodiodes as output detectors) were fabricated in the cleanroom facility of Fondazione Bruno Kessler (FBK). Two test PICs were fabricated and composed of Silicon Oxynitride SiON and non-stoichiometric SiN waveguides. One of the PICs included (Ti-TiN) thermistors as both heat-actuators and temperature-sensors for the phase shifters, while the other
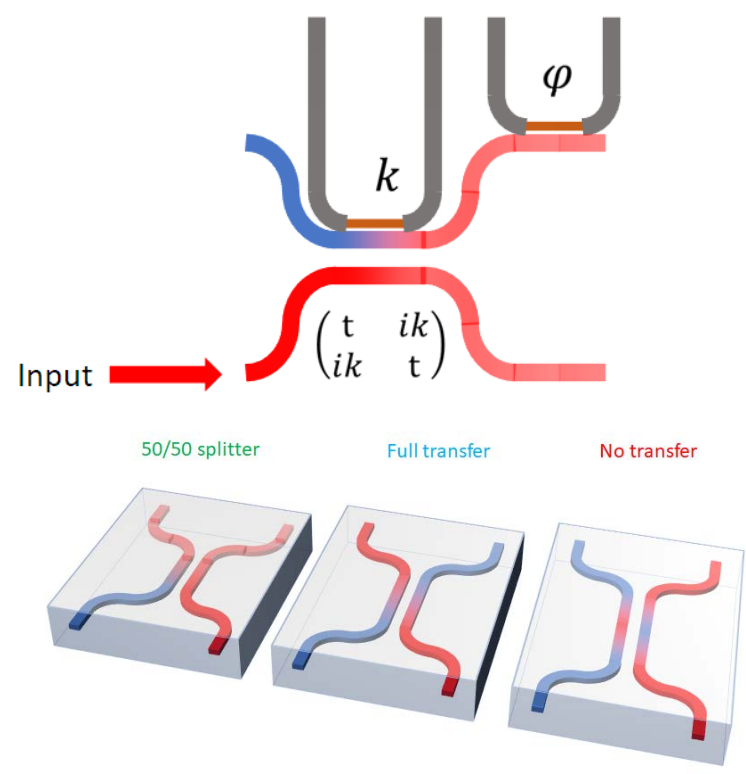

Figure 1: (top) schematics of Mach-Zehnder blocks and (bottom) main mode of operation of our directional coupler beam splitters. The induced phase shift $\varphi$ and split $k$ will be produced by Ti-TiN thermistors due to the thermo-optic effect.

one featured fully integrated silicon photodiodes meant to be used as output detectors. The material platform for the PIC-detector integrated circuits consists of silicon wafers with an epitaxial layer for the detectors. The detectors are realized by ion implantation and covered with a thick layer of TEOS glass to separate the PIC from the substrate. The PIC is realized in LPCVD and PECVD silicon nitride and oxynitride, respectively. The photonic layer is then covered again with a protective silicon oxyde to separate from the metal. The metal is realized with a combination of Ti, TiN and Al materials for the resistors, the contacts with the diode and the pads for wire-bonding. By combining beam splitters with phase shifters, up to $10 \mathrm{MZIs}$ need to be controlled with a total of 20 degrees of freedom, each controlled by a thermistor. To control thermistors, we will use a feedback loop algorithm, using the thermistors also as temperature sensors to better quantify the thermal field induced in the chip. We look forward to efficiently control the induced light change in the chip through Pulse-Width Modulation supply of the thermistors. An electronic control board is designed to fully monitor and control the PIC, with the capability to detect the thermistor maximum borne power and to measure the photodiode response to the photovoltaic excitation. In the following section, the characterization of the designed metallic thermistors, including the maximum borne power, are discussed.

\section{RESULTS}

We made electrical measurements of all integrated thermistors embedded in the PIC at a manual probe station at FBK. The probe 


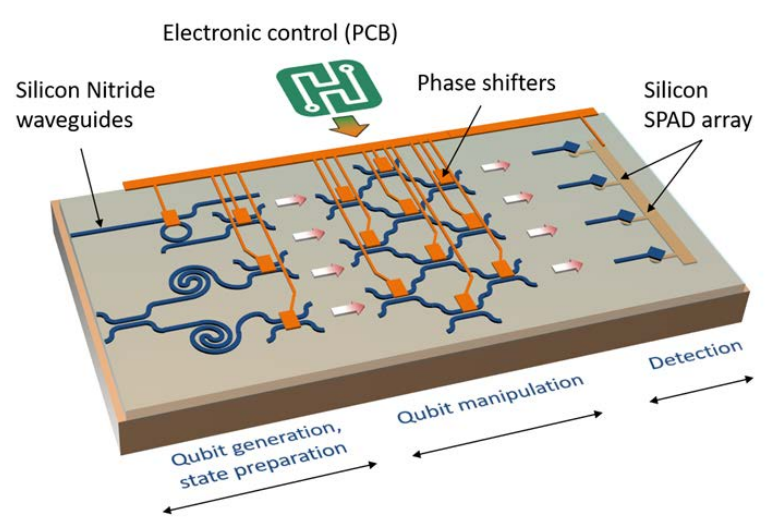

Figure 2: A sketch of the future PIC prototype. Through SiN waveguides we will guide an $850 \mathrm{~nm}$ laser beam and, by combining beam splitters and phase shifters, control $10 \mathrm{Mach}-$ Zehnder Interferometers to induce phase shifts. The output will be measured through an array of 6 silicon Single Photon Avalanche Detectors (SPADs). To fully monitor and control the PIC, an electronic control board will be designed, meeting the thermistor maximum borne power and the photodiode responde to the photovoltaic excitation.

station is equipped with a 200mm 4x PH150 Karl-Suss PM8 Manual Probe Station which ensured high stability and reliability of the measurement. Moreover, a LABVIEW interface was developed to collect the data. All the data were collected changing the potential incrementally from 0 to $15 \mathrm{~V}$. We produced I-V characterization curves for all the thermistors and computed the power consumption per unit length over potential and resistance values over dissipated power (Fig. 3). As shown in Fig. 4, we detected the maximum borne power density at $380 \mathrm{~W} / \mathrm{m}$, that is the highest power density that the smallest thermistor (namely the CPL2BA, with a length of $156 \mu \mathrm{m}$ ) can withstand before the breakage. This was reached at a potential of $12.4 \mathrm{~V}$. Regarding the resistance values, it can be noticed from Fig. 5 that all the ramp slopes are nearly identical, which is implied by having fixed cross-sections and same technology for all thermistors. In fact, because the slope represents the $\frac{d R}{d P}$ ratio, assuming a linear thermo-static model, this ratio corresponds to the $\frac{d R}{d T}$ ratio, meaning that an increase in power induces a linear increase in temperature. The same slope among all thermistors, which is $11000 \Omega / \mathrm{W}$, shows that the breakage load is the same for all thermistors having the same technology and cross-section. For the photodetectors (a close up on the first batch is presented in Fig. 6) one of the main parameters we will consider is the diode response to the photovoltaic excitation. As our involved power will be at the $\mu W$ level, a response in the order of $\mu A$ is expected. All the presented measurements are shown in plots below, as well as the thermistor lengths reported in table 1.

\section{CONCLUSIONS}

In this work, we studied and test control interfaces for PIC to C-MOS technology, to characterize the devices that will be used to fabricate

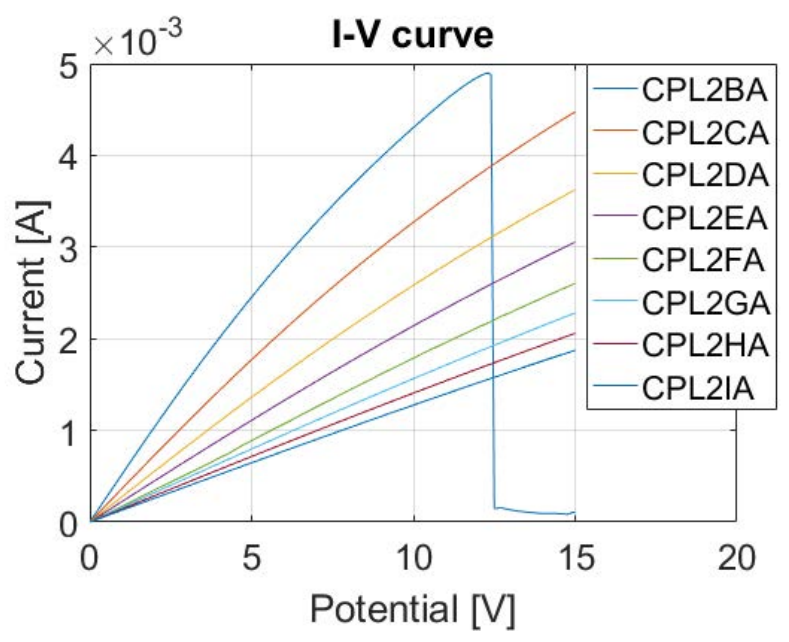

Figure 3: I-V characterization curves for thermistors. The blue line corresponds to the smallest thermistor, namely CPL2BA, which we pushed to breakage to estimate the maximum power per unit length obtainable (see next figure). The other lines correspond to longer resistances as described in Table 1

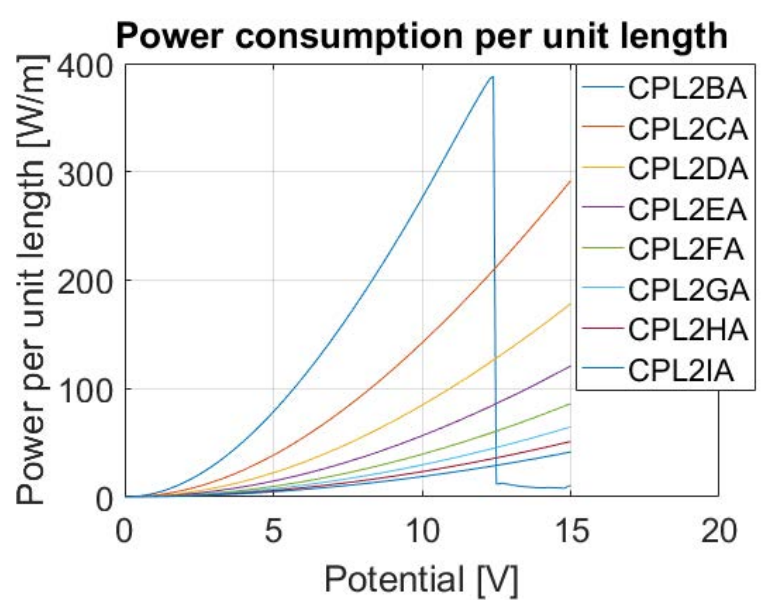

Figure 4: Power consumption per unit length for thermistors. We detected the breakage power density at about $\mathbf{3 8 0}$ $\mathrm{W} / \mathrm{m}$, that is the ratio between the dissipated power and the unit length immediately before component breakage occurred for the test resistance CPL2BA, with a length of $156 \mu m$.

prototypes of complex Photonic Integrated Circuits. Results showed that we were able to produce successfully and characterize resistive Ti-TiN thermistors as phase shifters for non-stoichiometric SiON optical waveguides. This set of experiments allowed us to identify the maximum borne power density for the thermistors realized with our microfabrication technology. The knowledge of this value is critical for the future design of an electronic circuit to control 


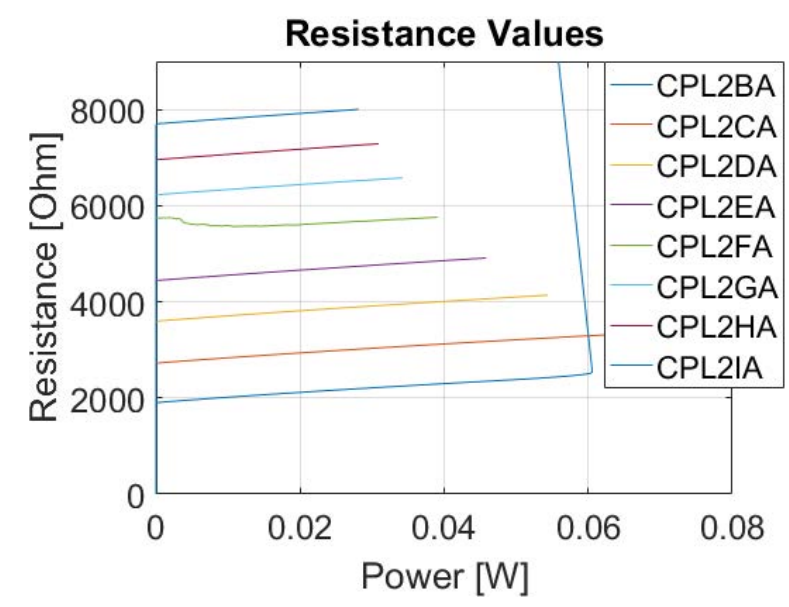

Figure 5: Resistance Values for thermistors. It is worth noticing that the slopes for each ramp is the same among all thermistors. That is because the slope represents the $\frac{d R}{d P}$ ratio, which, in a linear thermo-static model, is proportional to $\frac{d R}{d T}$ meaning that an increase in power induces a linear increase in temperature. The same slope among all thermistors, which is $11000 \Omega / \mathrm{W}$ thus shows that the breakage load is the same among all thermistors having the same technology and cross-section.

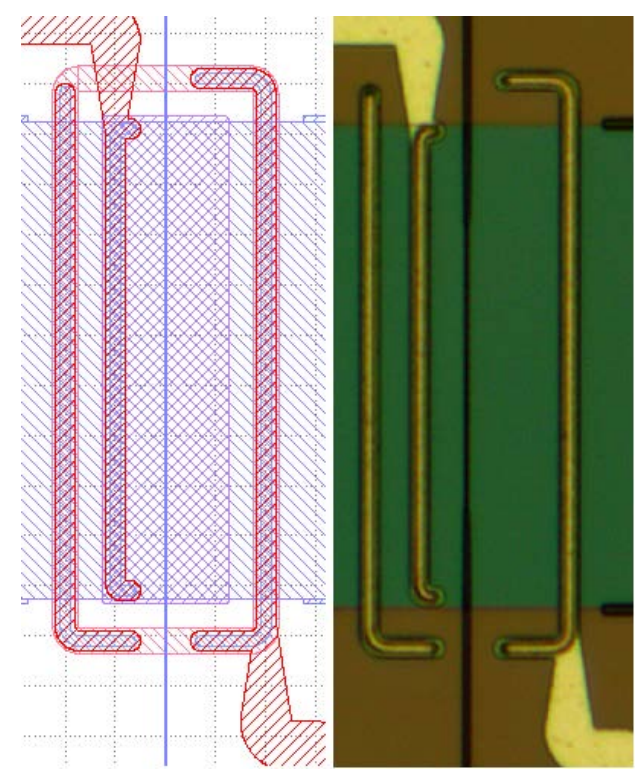

Figure 6: A close up on the designed photodetectors. (left) The CAD mask, (right) an optical micrograph of the fabricated device.

the PIC. Moreover, we validated the independence of the breakage load from the length. Future work will involve the full assessment of the photodiodes for a complete requirement definition of the electronic control scheme. In addition, we will design and test a
Table 1: Thermistor lengths

\begin{tabular}{c|c} 
Thermistor & Length $[\mu \mathrm{m}]$ \\
\hline CPL2BA & 156 \\
CPL2CA & 230 \\
CPL2DA & 305 \\
CPL2EA & 379 \\
CPL2FA & 454 \\
CPL2GA & 529 \\
CPL2HA & 603 \\
CPL2IA & 678
\end{tabular}

control system with feedback solutions, looking at both linear optic and quantum regimes. Future challenges will be the design and synchronization of the multichannel acquisition and reaching low latency in the response of the entire system.

\section{ACKNOWLEDGEMENTS}

We acknowledge the support of Giovanni Paternoster (FBK) for the design and realization of the photodiodes, as well as the support of the Microfabrication Laboratory staff of FBK during sample fabrication. We further acknowledge financial support from the Autonomous Province of Trento, under the initiative "Quantum at Trento - Q@TN", projects Q-PIXPAD and CoSiQuP. This project has received funding from the European Union's Horizon 2020 research and innovation programme under grant agreement No 777222, Attract INPEQuT.

\section{REFERENCES}

[1] The Verge. Google confirms 'quantum supremacy' breakthrough, 2019.

[2] The New York Times. Google claims a quantum breakthrough that could change computing, 2019.

[3] A. Annoni, E. Guglielmi, M. Carminati, S. Grillanda, P. Ciccarella, G. Ferrari, M. Sorel, M. J. Strain, M. Sampietro, A. Melloni, and F. Morichetti. Automated routing and control of silicon photonic switch fabrics. IEEE Journal of Selected Topics in Quantum Electronics, 22(6):169-176, Nov 2016.

[4] S. Zhu, T. Hu, Z. Xu, Y. Dong, O. Zhong, Y. Li, and N. Singh. An improved thermooptic phase shifter with aln block for silicon photonics. In 2019 Optical Fiber Communications Conference and Exhibition (OFC), pages 1-3, March 2019.

[5] A. A. Gentile, S. Paesani, R. Santagati, J. Wang, N. Wiebe, D. Tew, J. L. O’Brien, and M. G. Thompson. Noise resilience of bayesian quantum phase estimation tested on a si quantum photonic chip. In 2017 Conference on Lasers and Electro-Optics Europe European Quantum Electronics Conference (CLEO/Europe-EQEC), pages 1-1, June 2017.

[6] C. G. H. Roeloffzen, M. Hoekman, E. J. Klein, L. S. Wevers, R. B. Timens, D. Marchenko, D. Geskus, R. Dekker, A. Alippi, R. Grootjans, A. van Rees, R. M. Oldenbeuving, J. P. Epping, R. G. Heideman, K. Wörhoff, A. Leinse, D. Geuzebroek, E. Schreuder, P. W. L. van Dijk, I. Visscher, C. Taddei, Y. Fan, C. Taballione, Y. Liu, D. Marpaung, L. Zhuang, M. Benelajla, and K. Boller. Low-loss si3n4 triplex optical waveguides: Technology and applications overview. IEEE fournal of Selected Topics in Quantum Electronics, 24(4):1-21, July 2018. 